\title{
Efecto quimioprotector de Bidens pilosa en el cáncer de mama inducido en ratas
}

\author{
Bidens pilosa chemoprotective effect on induced breast cancer in rats
}

\author{
Jorge Arroyo ${ }^{1,2}$, Pablo Bonilla ${ }^{3}$, Ernesto Ráez ${ }^{4}$, Alejandro Barreda ${ }^{5}$, Oscar Huamán ${ }^{6}$ \\ ${ }^{1}$ Instituto de Investigaciones Clínicas, Facultad de Medicina, UNMSM. Lima, Perú. \\ 2 Departamento de Farmacología, Facultad de Medicina, UNMSM. Lima, Perú. \\ ${ }^{3}$ Instituto de Ciencias Farmacéuticas y Recursos Naturales, Facultad de Farmacia y Bioquímica, UNMSM. Lima, Perú. \\ ${ }^{4}$ Instituto de Patología, Facultad de Medicina, UNMSM. Lima, Perú. \\ ${ }^{5}$ Departamento Académico de Ginecología y Obstetricia, Facultad de Medicina, UNMSM. Lima, Perú. \\ ${ }^{6}$ Centro de Investigación en Bioquímica y Nutrición, Facultad de Medicina, UNMSM. Lima, Perú.
}

\begin{abstract}
Resumen
Introducción: Bidens pilosa L es una planta perteneciente a la familia Asteraceae, conocida en Perú como amor seco y cadillo. Se le atribuye efectos antiinflamatorio, diurético y hepatoprotector. Objetivos: Determinar el efecto quimioprotector de los compuestos fenólicos y flavonoides extraídos de la planta entera de Bidens pilosa sobre el cáncer de mama inducido en ratas con 7,12-dimetilbenz antraceno (DMBA). Protección medida en base a detención del desarrollo de adenocarcinoma y disminución de marcadores de estrés oxidativo. Diseño: Experimental. Institución: Laboratorio de Farmacología, Facultad de Medicina, Universidad Nacional Mayor de San Marcos. Material biológico: Planta entera de Bidens pilosa $L$ recolectada en La Libertad, Perú, y ratas hembras Holtzmann. Intervenciones: Se obtuvo los compuestos fenólicos y flavonoides por cromatografía en columna rápida, con solventes de polaridad creciente. Mediante cromatografía en capa fina y reactivos de desplazamiento, se aisló cuatro compuestos fenólicos. Los tumores de mama fueron inducidos con DMBA administrado oralmente. Se formó cuatro grupos de seis ratas cada uno: grupo control, grupo con tóxico inductor (TI) DMBA del cáncer, grupos con TI más tratamiento de extracto etanólico $300 \mathrm{mg} / \mathrm{kg}$, y grupo con TI más tratamiento de extracto metanólica $300 \mathrm{mg} / \mathrm{kg}$. Principales medidas de resultados: Disminución del desarrollo de adenocarcinoma mamario. Resultados: Se logró disminución significativa del desarrollo de adenocarcinoma mamario con los tratamientos de extracto metanólico y etanólico, siendo mejor con la fracción metanólica; el marcador de estrés oxidativo disminuyó en los grupos que recibieron tratamiento con la planta, siendo significativo con la fracción metanólica; hubo menor número de micronúcleos (genotoxicidad) en los animales que recibieron tratamiento. Conclusiones: En condiciones experimentales, el extracto y fracción metanólica de Bidens pilosa detuvieron la progresión del cáncer mamario inducido en ratas y disminuyeron el estrés oxidativo.
\end{abstract}

Palabras clave: Cáncer mama; Bidens pilosa; compuestos fenólicos.

\begin{abstract}
Introduction: Bidens pilosa species belonging to the Asteraceae family, known in Peru as love dry bur, is credited with anti-inflammatory, diuretic, hepatoprotective effects. Objectives: To determine the protective effect of phenolic compounds and flavonoids extracted from Bidens pilosa $L$ whole plant on breast cancer induced in rats by 7,12-dimethylbenz (A) anthracene (DMBA). Design: Experimental. Setting: Laboratory of Pharmacology, Faculty of Medicine, Universidad Nacional Mayor de San Marcos, Lima, Peru. Biological material: Bidens pilosa L whole plant collected in Santiago de Cao, Ascope, La Libertad, and female rats. Interventions: Phenolic compounds and flavonoids were obtained by rapid column chromatography with solvents of increasing polarity; by thin layer chromatography on a preparative scale four phenolic compounds were isolated and elucidated by UV-Visible spectroscopy with shift reagents. Breast tumors were induced with DMBA administered orally to four groups of rats: normal control group, group with toxic inductor (TI) of cancer, group with $\mathrm{TI}$ and ethanolic extract, and group with $\mathrm{TI}$ and methanol fraction in doses of $300 \mathrm{mg} / \mathrm{kg}$. Main outcome measures: Induced breast cancer protection. Results: Development of mammary adenocarcinoma tends to slow with treatments, more with the methanol fraction; the oxidative stress marker decreased in the groups treated with the plant, better with the methanol fraction; there were fewer micronuclei (genotoxicity) in animals receiving treatment. Conclusions: Under experimental conditions both Bidens pilosa methanol extracts and fractions halted induced breast cancer progression in rats.
\end{abstract}

Key words: Breast neoplasms; Bidens pilosa; phenolic compounds.

An Fac med. 2010;71(3):153-9

\section{INTRODUCCIÓN}

Los flavonoides contienen en su estructura química un número variable de grupos hidroxilo fenólicos con excelentes propiedades de quelación del hierro y otros metales de transición, lo que les confiere una gran capacidad antioxidante ${ }^{(1)}$. Por ello, desempeñan un papel esencial en la protección frente a los fenómenos de daño oxidativo y tienen efectos terapéuticos en diversas patologías, incluyendo la cardiopatía isquémica, la aterosclerosis y el cáncer ${ }^{(2)}$. Los compuestos fenólicos y flavonoides son pigmentos naturales presentes en los vegetales y que protegen al organismo del daño producido por agentes oxidantes; están ampliamente distribuidos en plantas, frutas, verduras y representan componentes sustanciales de la parte no energética de la dieta humana ${ }^{(3)}$. Su estructura química les permite ser atrapadores de radicales libres, desempeñando así un importante rol antioxidante en patologías relevantes en la salud pública como el cáncer ${ }^{(1,2)}$.
El estudio fitoquímico de los extractos y fracciones de la planta total de Bidens pilosa L (conocida popularmente como 'amor seco') evidencia la presencia de chalconas, glucósidos del ácido fenilpropanoico, poliacetilenos, un diterpeno, flavonoides, glucósidos de flavona y compuestos fenólicos ${ }^{(4)}$. Asimismo, en estudios previos se ha determinado las siguientes propiedades de Bidens pilosa L: antihipertensiva ${ }^{(5)}$, antiulcerosa ${ }^{(6)}$, hepatoprotectora (7), inmunosupresora y antiinflamatoria ${ }^{(8)}$, antileucémica ${ }^{(9)}$, 
antimicrobiana ${ }^{(10)}$ y quimioprotectora de neoplasia gástrica ${ }^{(11)}$.

Así, la presente investigación tuvo por objetivo obtener la fracción que contiene compuestos fenólicos y flavonoides de la planta entera de Bidens pilosa L para determinar su efecto quimioprotector sobre el cáncer de mama inducido en ratas, estableciendo su efecto antigenotóxica mediante un marcador de estrés oxidativo, dado el demostrado efecto antioxidante de esta planta y a que el estrés oxidativo es considerado, en la actualidad, parte importante en la patogénesis del cáncer.

\section{MÉTODOS}

Se realizó un estudio de tipo experimental, en el que se usó 24 ratas (4 grupos de 6 ratas) albinas de la cepa Holtzman, con peso al inicio de la investigación de 100 a $130 \mathrm{~g}$, de 2 meses de edad, hembras, procedentes del Bioterio del Instituto Nacional de Salud del Ministerio de Salud, mantenidas en un ambiente a temperatura de $21^{\circ} \mathrm{C}$, con dieta y agua a libertad. La planta Bidens pilosa L. ('amor seco') fue recolectada en el caserío de San José Bajo, distrito de Santiago de Cao, provincia de Ascope, departamento de la Libertad, Perú. Como inductor de cáncer de mama se utilizó 7,12-dimetilbenz (A) antraceno (DMBA) sigma. Como solventes se utilizó alcohol 96 (etanol), metanol A.C.S (Fisher), N-hexano P.A (Merck), cloroformo ACS-HPLC (Fisher), Silicagel para cromatografía en columna rápida, hidróxido de sodio 0,5 N, EDTA, formol $40 \%$, heparina sódica, colorante Giemsa 0,6\%, aceite de inmersión.

\section{Obtención de compuestos fenólicos y flavonoides}

La obtención de la fracción que contiene compuestos fenólicos y flavonoides de la planta entera de Bidens pilosa L se realizó según la siguiente secuencia: ubicación y recolección de la muestra vegetal, clasificación taxonómica, molienda y almacenamiento, preparación del extracto etanólico siguiendo parámetros establecidos ${ }^{(11-14)}$, cromatografía en columna rápida (para lo cual se utilizó solventes de polaridad creciente: n-hexano, cloroformo, metanol y agua destilada, con el fin de fraccionar el extracto etanólico total y separar metabolitos afines para poder relacionar estructura química con actividad farmacológica de cada subextracto, obteniéndose así: fracción N-hexano, fracción clorofórmica, fracción metanólica, fracción etanólica y fracción acuosa) y finalmente se realizó una marcha fitoquímica ${ }^{(13)}$. Para la mencionada marcha fitoquímica, cada reacción de identificación de metabolitos secundarios presentes en el extracto etanólico se realizó con los reactivos específicos, reportando los resultados como presencia o ausencia del metabolito ( $5 \mathrm{mg}$ de extracto problema con 5 gotas de reactivos). Del extracto etanólico de Bidens pilosa mediante cromatografía en capa fina a escala preparativa se aisló cuatro compuestos fenólicos, los que fueron elucidados mediante espectroscopia de luz uv-visible y con reactivos de desplazamiento. Se utilizó un espectrofotómetro de marca Hewlett Packard modelo 8452 A Diode array, con rango de longitud de onda entre 200 y $800 \mathrm{~nm}$ y las lecturas fueron realizadas con una longitud de onda de 200 a $400 \mathrm{~nm}$.

La inducción del cáncer de mama consistió en administrar $20 \mathrm{mg}$ de 7,12. dimethylbenz (A) antraceno (DMBA), por única vez, vía oral, diluido en $1 \mathrm{~mL}$ de aceite de oliva ${ }^{(15)}$. Veinticuatro ratas hembras fueron divididas en cuatro grupos de seis cada uno: el primer grupo recibió suero fisiológico $5 \mathrm{~mL} / \mathrm{kg}$ (control negativo); el segundo grupo recibió DMBA (control positivo); al tercer y cuarto grupos, además de DMBA, se les administró extracto alcohólico y fracción metanólica de Bidens pilosa, respectivamente, en dosis de $300 \mathrm{mg} / \mathrm{kg}$, vía oral (VO). Luego de 14 semanas, los animales fueron sacrificados. Se evaluó las mamas macro y microscópicamente, las mismas que fueron conservadas en formol al 10\% para efectuar el estudio histopatológico, donde se determinó el tipo y forma de células tumorales.

La actividad antigenotóxica se midió por medio de la prueba de micronúcleos ${ }^{(16)}$. Se extrajo sangre por punción cardiaca y una gota fue extendida sobre una lámina portaobjetos, luego fueron fijadas con metanol y teñidas en solu- ción Giemsa a 3\%. Se contabilizó 100 eritrocitos, anotándose los micronúcleos encontrados (porcentaje de células micronucleadas), los eritrocitos policromáticos (PCE) y eritrocitos normocromáticos (NCE). Debido a la presencia del tóxico inductor del cáncer, el efecto antigenotóxico fue determinado por la frecuencia de eritrocitos policromáticos micronucleados presentes.

La determinación del marcador de estrés oxidativo ${ }^{(17,18)}$ fue realizada en suero procedente de la sangre extraída de las ratas sometidas a inducción del cáncer de mama. Se efectuó la prueba de lipoperoxidación, que implica la conversión oxidativa de ácidos grasos insaturados (hidroperóxidos lipídicos o lipoperóxidos). La lipoperoxidación fue medida mediante la producción de malondialdehído, que al reaccionar con el ácido tiobarbitúrico forma un complejo coloreado que fue leído a $535 \mathrm{~nm}$, siendo expresados en número de moles de malonaldialdehído x $10^{-6}$.

La eficacia quimioprotectora fue analizada mediante la prueba del chi cuadrado, la prueba de probabilidad exacta de Fisher y análisis de varianza con múltiples comparaciones de Duncan. Los datos de micronúcleos y el nivel del marcador de estrés oxidativo fueron evaluados mediante análisis de varianza y media de grupos comparadas con la prueba de LSD (diferencia significativa mínima). Para los diferentes análisis, se consideró valor de $p<0,05$. Se utilizó el programa estadístico SPSS versión 13.

\section{RESULTADOS}

En el estudio fitoquímico de Bidens pilosa, se obtuvo un extracto etanólico con aspecto de masa homogénea, consistencia blanda, color verde petróleo, libre de partículas extrañas. El rendimiento fue de $9,4 \%$ de la planta entera. El estudio fitoquímico mostró que los flavonoides, compuestos fenólicos y taninos estuvieron en mayor cantidad en la fracción metanólica (tabla 1).

Para la cromatografía en capa delgada a escala preparativa, se preparó cromatoplacas de 20 x $20 \mathrm{~cm}$ de longitud, con 
Tabla 1. Estudio fitoquímico de Bidens pilosa L.

\begin{tabular}{llccccc}
\hline Reacción de: & \multicolumn{1}{c}{$\begin{array}{c}\text { Metabolitos } \\
\text { secundarios }\end{array}$} & $\begin{array}{c}\text { Extracto } \\
\text { etanólico }\end{array}$ & $\begin{array}{c}\text { Fracción } \\
\text { metanol }\end{array}$ & $\begin{array}{c}\text { Fracción } \\
\text { cloroformo }\end{array}$ & $\begin{array}{c}\text { Fracción } \\
\text { N-hexano }\end{array}$ & $\begin{array}{c}\text { Fracción } \\
\text { acuosa }\end{array}$ \\
\hline Dragendorf & Alcaloides & - & - & - & - & - \\
Shinoda & Flavonoides & ++ & ++ & + & - & - \\
Espuma & Saponinas & - & - & - & - & - \\
Tricloruro férrico & Compuestos fenólicos & ++ & +++ & + & + & + \\
Gelatina & Taninos & ++ & ++ & + & - & - \\
Lieberman-Burchard & Esteroides ylo terpenoides & - & - & - & - & - \\
Ninhidrina & Aminoácidos libres & - & - & - & - & - \\
Molish (alfa naftol) & Glicósidos & ++ & ++ & - & - & - \\
\hline
\end{tabular}

Ausencia (-) $\quad$ Leve cantidad (+) Regular cantidad (++) $\quad$ Abundante cantidad (+++)

sílica gel G-60. Se sembró la muestra de Bidens pilosa en cinco carriles, de manera vertical. Luego, la placa sembrada se desarrolló en una cuba que contenía el sistema de solventes: diclorometano y metanol (9:1). Luego del corrido del solvente hasta el frente de solventes, se procedió a secar la placa y luego al revelado con la luz uv a 365 y $254 \mathrm{~nm}$. La marcha fitoquímica permitió establecer que Bidens pilosa contiene principalmente compuestos fenólicos (flavonoides, taninos, etc.) y alcaloides. Los cromatogramas en capa fina del extracto demostraron que

contenía gran cantidad de compuestos fenólicos y flavonoides, siendo los derivados metoxi flavona los sobresalientes (figuras 1 y 2 ).

Con relación a la elucidación estructural, se propone estructuras químicas al realizar el análisis del comportamiento químico y fisicoquímico ${ }^{(12,13)}$ por medio de los espectros ultravioleta visibles en metanol y sus correspondientes reacciones de desplazamiento de las fracciones aisladas mediante técnicas cromatográficas en capa fina a escala preparativa del extracto de Bidens pilosa:

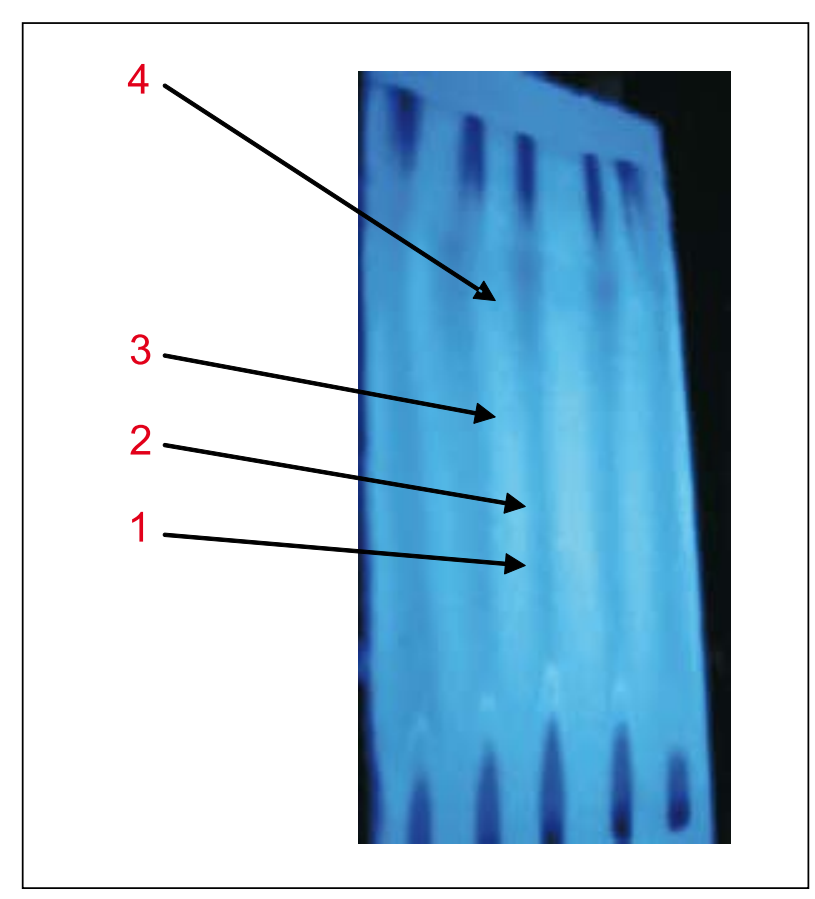

Figura 1: Cromatografía en capa delgada a escala preparativa del extracto de Bidens pilosa, a la luz uv 365nm.
1. Fracción etanólica I ( MI ). Lecturas obtenidas: uv-visible:

máx. $\lambda^{\mathrm{MeOH}}: 270,330 \mathrm{~nm}$

máx. $\lambda^{\mathrm{MeOH}+\mathrm{MeO} \mathrm{Na}}: 275,330 \mathrm{~nm}$

máx. $\lambda^{\mathrm{MeOH}+\mathrm{AlCl}_{3}}: 275,330 \mathrm{~nm}$

máx. $\lambda^{\mathrm{MeOH}+\mathrm{AlCl}_{3}+\mathrm{HCl}}: 275,330 \mathrm{~nm}$

máx. $\lambda^{\mathrm{MeOH}+\mathrm{AcONa}}: 275,330 \mathrm{~nm}$

Comparando y analizando los espectros uv-visible desarrollados y sus correspondientes reacciones de desplazamiento con los reportados por Mabry ${ }^{(12,13)}$, esta fracción corresponde al 5,7-dihidroxi-4'metoxi flavona.

2. Fracción etanólica II (MII). Lecturas obtenidas: uv-visible.

máx. $\lambda^{\mathrm{MeOH}}: 255,260,314 \mathrm{~nm}$

máx. $\lambda^{\mathrm{MeOH}+\mathrm{MeO} \mathrm{Na}}: 255,260,314 \mathrm{~nm}$

máx. $\lambda^{\mathrm{MeOH}+\mathrm{AlCl}_{3}}: 255,260,314 \mathrm{~nm}$

máx. $\lambda^{\mathrm{MeOH}+\mathrm{AlCl}_{3}+\mathrm{HCl}}: 255,260,314 \mathrm{~nm}$

máx. $\lambda^{\mathrm{MeOH}+\mathrm{AcONa}}: 255,260,314$ nm

Comparando y analizando los datos anteriores de los espectros uv-visible y sus correspondientes reacciones de desplazamiento con los reportados por Mabry ${ }^{(12,13)}$, se propone la siguiente estructura: 4' - hidroxi-7-metoxi isoflavona.

3. Fracción etanólica III (MIII). Lecturas obtenidas: uv-visible.

máx. $\lambda^{\mathrm{MeOH}}: 254,270,315 \mathrm{~nm}$

máx. $\lambda^{\mathrm{MeOH}+\mathrm{MeO} \mathrm{Na}}: 254,270,315 \mathrm{~nm}$

máx. $\lambda^{\mathrm{MeOH}+\mathrm{AlCl}_{3}}: 260,275,315 \mathrm{~nm}$

máx. $\lambda^{\mathrm{MeOH}+\mathrm{AlCl}_{3}+\mathrm{HCl}}: 260,275,315 \mathrm{~nm}$

máx. $\lambda^{\mathrm{MeOH}+\mathrm{Ac} \mathrm{ONa}}: 255,260,314$ nm (hay descomposición)

Al analizar y comparar los datos obtenidos de los espectros uv-visible y sus correspondientes reacciones de desplazamiento con los reportados por Mabry ${ }^{(12,13)}$, se propone la siguiente estructura: 5 - hidroxi-4', 7-dimetoxi flavona.

4. Fracción etanólica IV (MIV). Lecturas obtenidas: uv-visible.

máx. $\lambda^{\mathrm{MeOH}}: 255,275,310 \mathrm{~nm}$

máx. $\lambda^{\mathrm{MeOH}+\mathrm{MeO} N a}: 255,275,310 \mathrm{~nm}$

máx. $\lambda^{\mathrm{MeOH}+\mathrm{AlCl}_{3}}: 265,275,310 \mathrm{~nm}$

máx. $\lambda^{\mathrm{MeOH}+\mathrm{AlCl}_{3}+\mathrm{HCl}}: 265,275,310 \mathrm{~nm}$ 


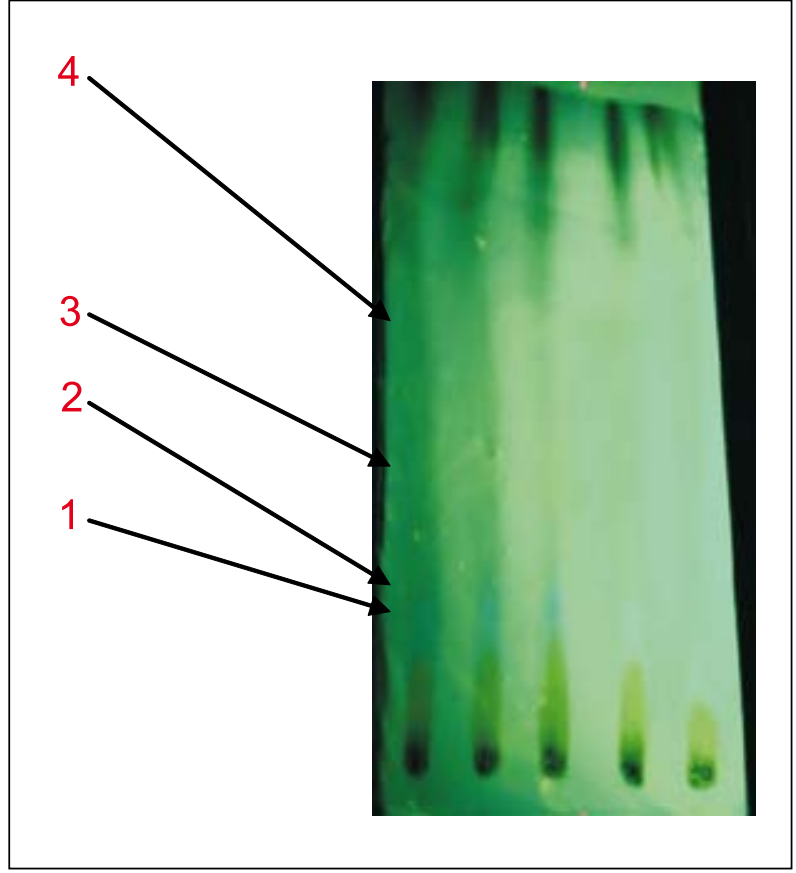

Figura 2: Cromatografía en capa delgada a escala preparativa del extracto de Bidens pilosa, a la luz uv $254 \mathrm{~nm}$.

Al comparar y analizar los datos obtenidos de los espectros uv-visible y sus correspondientes reacciones de desplazamiento con los reportados por Mabry ${ }^{(12,13)}$, se propone la siguiente estructura: 5 - hidroxi- 7- metoxi flavona.

Sobre la eficacia quimioprotectora de la fracción rica en compuestos fenólicos de Bidens pilosa L, en la tabla 2 se aprecia la respuesta sobre los adenocarcinomas de mama por efecto del extracto etanólico y fracción metanólica de Bidens pilosa, observándose un mejor efecto con la fracción metanólica. La figura 3 muestra las características de un adenocarcinoma mamario intraductal en el grupo que solo recibió DMBA. Las figuras 4 y 5 muestran las respuestas favorables del extracto etanólico y metanólico de Bidens pilosa sobre el cáncer de mama inducido en ratas. En la figura 6 se aprecia un gráfico de barras con las características histopatológicas, observándose una mayor alteración en el grupo que solo recibió DMBA, mientras que en los grupos tratados con extracto etanólico y fracción metanólica hubo una disminución, destacando el grupo que recibió fracción metanólica.

Respecto al ensayo de antigenotoxicidad, en la tabla 3 se observa la presencia de eritrocitos policromados micronucleados en mayor cantidad en las ratas que recibieron tóxico inductor solamente, mientras que en aquellas que recibieron

Tabla 2. Observaciones histopatológicas agrupadas según tratamientos, en la inducción del cáncer de mama en ratas.

\begin{tabular}{|c|c|c|}
\hline$N^{0}$ & Tratamiento & Observaciones de todas las ratas por grupo $(n=6)$ \\
\hline 1 & Control (-) normal & Sin alteraciones significativas \\
\hline 2 & Control (+) tóxico inductor (TI) & $\begin{array}{l}\text { Se llega a formar neoplasia maligna metastásica, } \\
\text { adenocarcinoma mamario intraductal (++++) }\end{array}$ \\
\hline 3 & $\mathrm{TI}+$ extracto etanólico de Bidens pilosa $\mathrm{L}$ & Adenocarcinoma diferenciado (++), desmoplásico \\
\hline 4 & $\mathrm{TI}$ + fracción metanólica de Bidens pilosa L & La sustancia impidió mayor diferenciación \\
\hline
\end{tabular}

tóxico inductor más extracto etanólico y fracción metanólica mostraron disminución de eritrocitos policromados micronucleados, siendo mejor en aquellas que recibieron fracción metanólica.

\section{Marcador de estrés oxidativo}

La figura 7 muestra que el nivel de radicales libres fue mayor para el grupo que recibió solo DMBA, mientras que el extracto etanólico mostró un mayor efecto que la fracción metanólica

\section{DISCUSIÓN}

El 7,12-dimetilbenz (A) antraceno (DMBA) es uno de los hidrocarburos policiclícos aromáticos más potentes para inducir cáncer de mama, debido a su bioactivación e interacción con el ADN. Así, en la célula mamaria, el metabolito reactivo diol-epóxido adiciona residuos de adenina y guanina al ADN. La formación de metabolitos genotóxicos como el diol epóxido del DMBA es favorecida por acción de la familia de citocromo P450. Se ha identificado CYP1A1 y CYP1B1 como enzimas que metabolizan la conversión del DMBA a 3,4-diol-epóxido. $\mathrm{Al}$ respecto, se conoce que los flavonoides tipo naftoflavona, inhiben competitivamente CYP1A y además inhiben la unión al ADN (19-21). En nuestro estudio, los cromatogramas en capa fina del extracto demostraron contener gran cantidad de compuestos fenólicos y flavonoides, siendo los derivados metoxi flavona los sobresalientes.

Por otro lado, los flavonoides de las plantas han mostrado actividad antiinflamatoria in vitro e in vivo ${ }^{(22)}$. Uno de estos mecanismos es por inhibición de eicosanoides generadores de enzimas, incluyendo fosfolipasa $\mathrm{A} 2$, ciclooxigenasa y lipoxigenasa, reduciendo así la presencia de prostanoides y leucotrienos. Además, recientes estudios muestran que los flavonoides, especialmente flaconas y sus derivados, expresan una gran actividad antiinflamatoria debido a la modulación de la expresión de genes proinflamatorios, tales como ciclooxigenasa-2, óxido nítrico sintasa inducible y varias citoquinas pivotales ${ }^{(23,24)}$. 


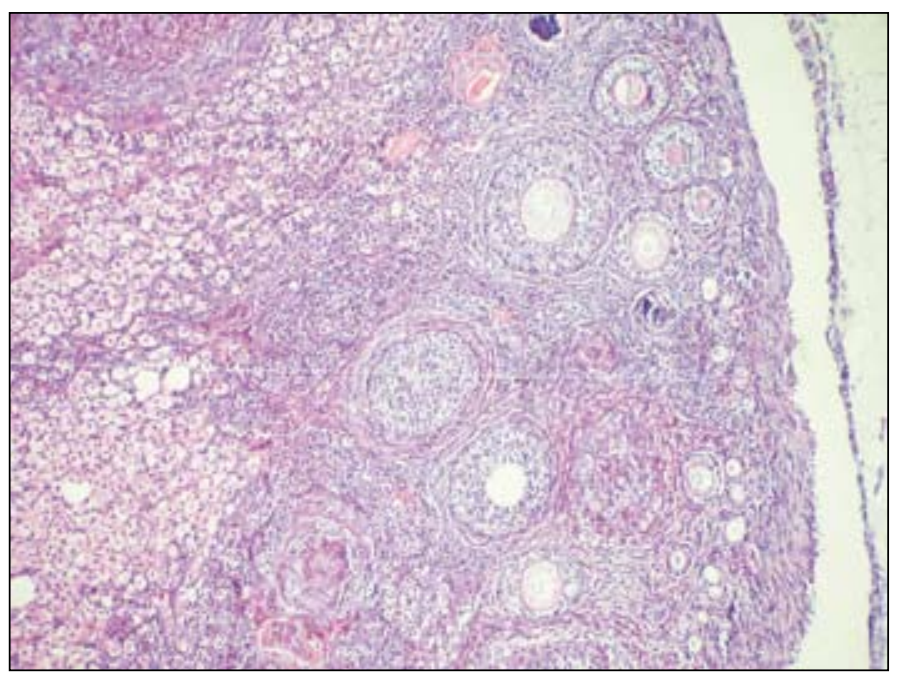

Figura 3. Adenocarcinoma mamario intraductal. 100X.

En las ratas, a la edad de 45 y 60 días se inicia la organogénesis activa en la glándula mamaria, con alto índice de la proliferación de lóbulos tipos 1 y 2; por ello se recomienda la administración del DMBA en esta fase de la vida de los animales para la inducción del cáncer de mama. Los tumores inducidos son generalmente carcinomas ductal o carcinomas papilares, pero es posible que fibroadenomas, adenomas y papilomas típicos también se formen. La proliferación epitelial y mioepitelial de la célula fue observada en la mayoría de los tumores inducidos en nuestro experimento, tal como se ha referido también en otros estudios ${ }^{(25)}$. La mayoría de lesiones encontradas en ratas tiene correspondencia con las de seres humanos, permitiendo la extrapolación de lo experimental a lo clínico.

El DMBA es altamente lipofílico y requiere la activación metabólica para su carcinogenicidad. Varios tejidos son capaces de activar DMBA, entre los que se incluye la glándula mamaria; en dicho tejido, el DMBA se convierte en metabolitos epóxidos con gran capacidad de dañar el ADN, lo que constituye el evento principal en la iniciación de la

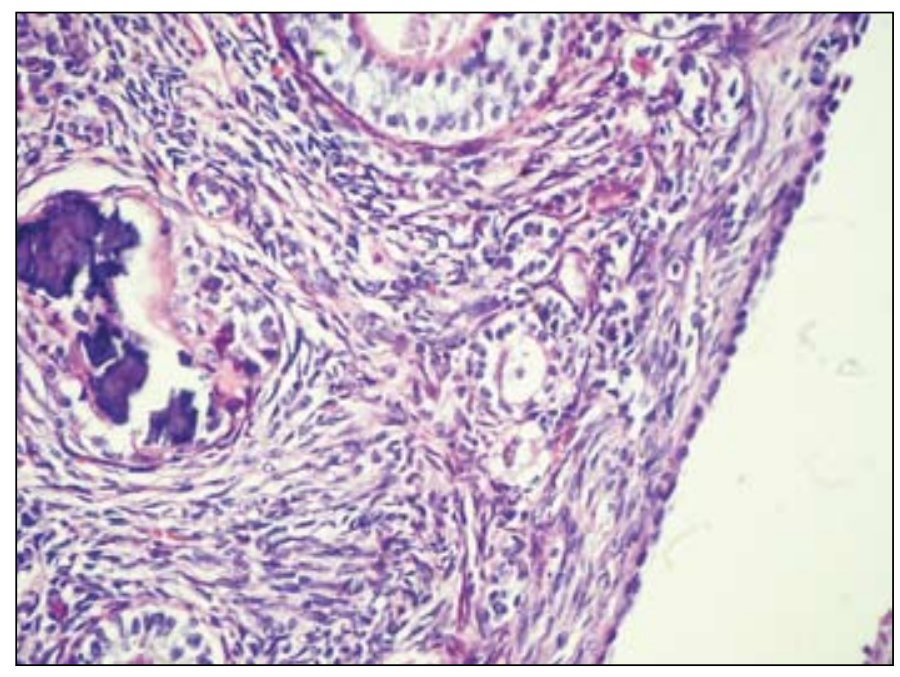

Figura 4. Efecto del extracto etanólico de Bidens pilosa L. 100X. Reducción discreta del tamaño de los conductos superficiales, infiltración de células neoplásicas con una zona de necrosis central. carcinogénesis. Los lóbulos 1 y 2 de la glándula mamaria presentan el índice proliferativo celular más alto y por tanto tienen más actividad metabólica; esto a su vez genera más producción de epóxido y radicales libres ${ }^{(26)}$. Por otro lado, se conoce que los estrógenos son responsables de la iniciación y promoción del cáncer de mama; así, la inhibición de la síntesis de estrógenos por luteína (4-tetrahidrochalcona) puede ser efectiva en la prevención del cáncer de mama, al inhibir fuertemente la aromatasa ${ }^{(27)}$. Posiblemente, algunas sustancias presentes en el estudio fitoquímico de Bidens pilosa, como las chalconas, hayan favorecido los hallazgos obtenidos en el estudio ${ }^{(4)}$.

Las especies reactivas del oxígeno (ROS), como el superóxido, el peróxido de hidrógeno, el oxhidrilo, el óxido nítrico y radicales del peroxinitrito, desempeñan un papel importante en el estrés oxidativo relacionado con la patogenia de varias enfermedades. En individuos sanos, la producción de radicales libres es balanceada por el sistema antioxidante de la defensa; sin embargo, se genera estrés oxidativo cuando el equilibrio favorece la generación del radical libre, como resultado de un agotamiento de niveles antioxidantes. El daño oxidativo, causado por la acción de radicales libres, puede iniciar y promover la progresión de algunas enfermedades crónicas, tales como cáncer, enfermedades cardiovasculares, desórdenes neurodegenerativos y envejecimiento ${ }^{(28,29)}$. Según nuestros resultados, los tratamientos administrados en la inducción de cáncer de mama muestran una menor cantidad de moléculas del complejo coloreado malondialdehido ácido tiobarbitúrico, lo que indica una disminución de radicales libres y por tanto un menor daño de la membrana celular (menor lipoperoxidación de la membrana celular).

Nuestro hallazgo principal fue la reducción de casos de cáncer, debido a los flavonoides de Bidens pilosa, cuyo beneficio puede explicarse porque los flavonoides inducen apoptosis al activar la caspasa 8 y Bax, inhiben la expresión del Bcl-2 y permiten la liberación de citocromo $\mathrm{C}^{(30)}$. También, los flavonoides poseen actividad antioxidante y antiangiogéni- 


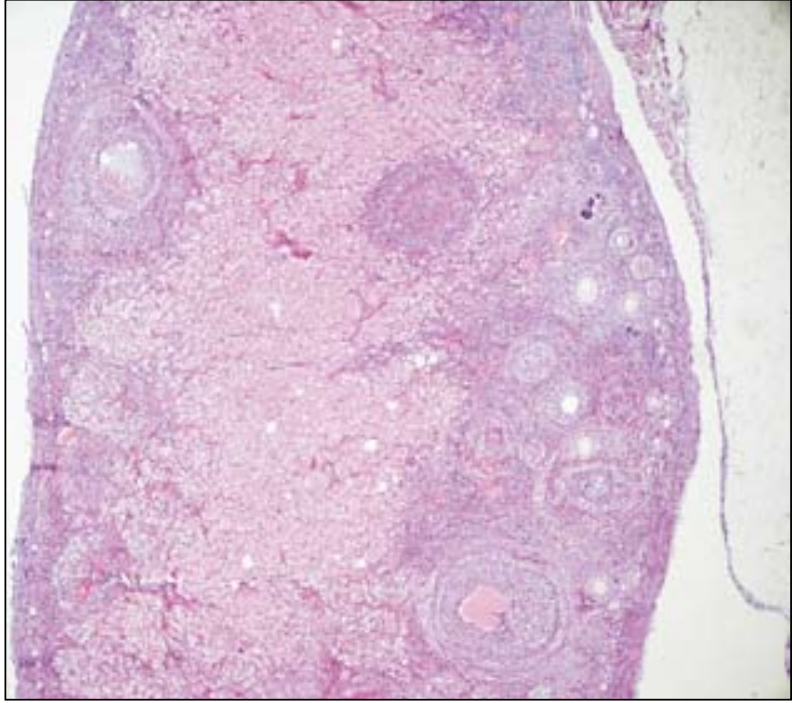

Figura 5. Efecto de la fracción metanólica de Bidens pilosa L. 100X. Reducción del número de conductos con proliferación cancerosa, glándula mamaria con infiltración carcinomatosa dentro de los conductos, desmoplasia.

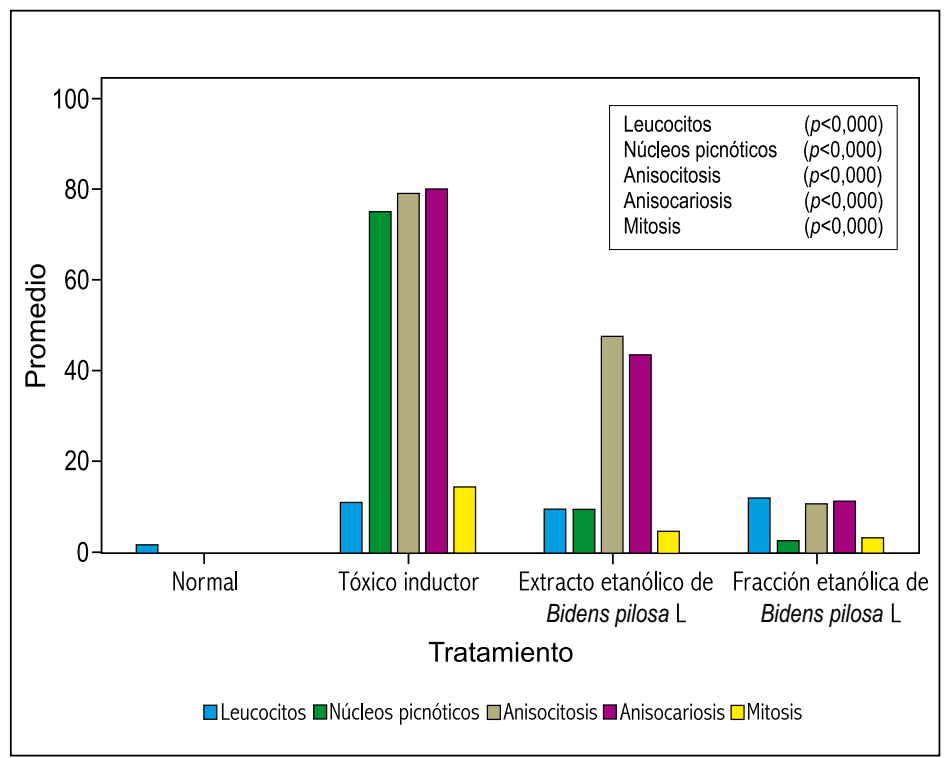

Figura 6. Características histopatológicas en la inducción de cáncer de mama en ratas.

Tabla 3. Ensayo de antigenotoxicidad mediante búsqueda de micronúcleos en sangre de ratas con inducción de cáncer de mama.

\begin{tabular}{lccc}
\hline \multicolumn{1}{c}{ Tratamiento } & PCE & mPCE & \% dism. mPCE \\
\hline Control (-) Normal & 1122 & 0,3 & 0 \\
Control (+) Tóxico inductor (TI) & 3147,32 & 3,25 & 100 \\
TI + Extracto etanólico de Bidens pilosa L & 1606,5 & 1,52 & 58,2 \\
TI + Fracción metanólica de Bidens pilosa L & 1470,5 & 1,05 & 67,7 \\
\hline
\end{tabular}

$\mathrm{PCE}=$ eritrocitos policromáticos normales; $\mathrm{mPCE}=$ eritrocitos policromados micronucleados co, al suprimir la proliferación del factor de crecimiento vascular (VEGF) ${ }^{(31)}$. Por otro lado, existe información científica que estos podrían inducir riesgos de mutagenotoxicidad ${ }^{(32)}$; pero, según la tabla 3 se muestra que la influencia de Bidens pilosa $\mathrm{L}$ reduce el nivel de micronúcleos, que se relaciona con una menor genotoxicidad.

El cáncer mama es un problema de salud pública, ocupa el segundo lugar de mortalidad en mujeres a partir de 20 años en el Perú ${ }^{(33)}$, y entre el primer y segundo lugar a nivel internacional ${ }^{(4)}$. Debido a la resistencia farmacológica y los marcados efectos adversos de los agentes que actualmente se emplea en su tratamiento, se hace necesaria la búsqueda de nuevos productos, de preferencia naturales, que puedan demostrar eficacia y mínimos efectos adversos en el manejo de esta enfermedad. Es por ello que consideramos valiosos los aportes de la presente investigación, que servirá para futuros ensayos sobre los flavonoides de Bidens pilosa, como agentes con potencial reductor de la progresión del cáncer mamario.

En conclusión, en condiciones experimentales se ha demostrado que el extracto y fracción metanólica de Bidens pilosa $\mathrm{L}$ detuvieron la progresión del cáncer mamario inducido en ratas.

\section{Agradecimientos}

Al Consejo Superior de Investigaciones de la Universidad Nacional Mayor de San Marcos por el financiamiento del Proyecto FEDU de código 060103131, año 2006.

\section{REFERENCIAS BIBLIOGRÁFICAS}

1. Haysteen B. Flavonoids. A class of natural products of high pharmacological potency. Biochem Pharmacol. 1983;32:1141-8.

2. Pace-Asciak C, Hahn S, Diamandis E, Soleas $G$, Goldberg D. The red wine phenolics transresveratrol and quercitin block human platelet aggregation in eicosanoid synthesis: implication for protection against coronary heart disease. Clin Chim Acta. 1995;235:207-19.

3. Aherne S, O'Brien N. Dietary flavonols: chemistry, food content, and metabolism. Nutrition. 2002;18:75-81. 


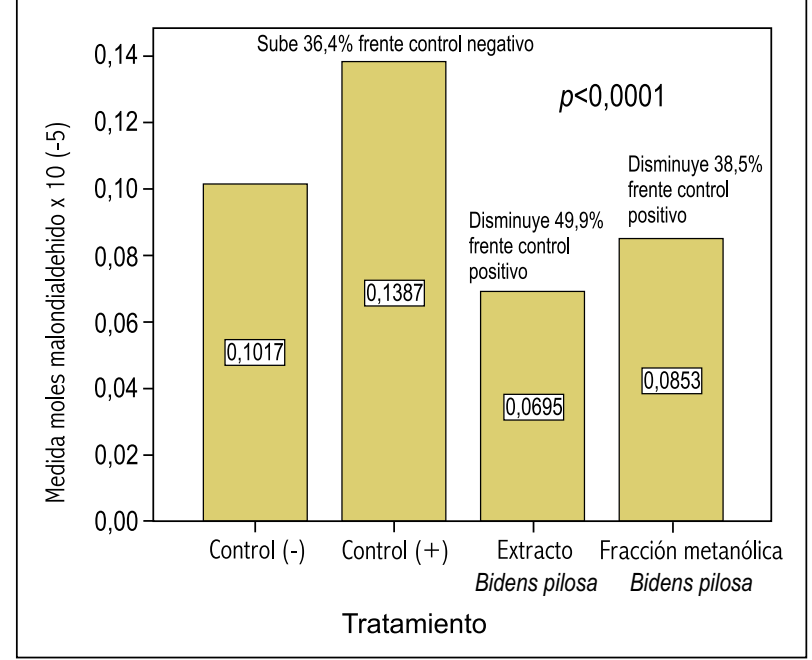

Figura 7. Nivel de lipoperoxidación en suero de ratas con inducción de cáncer de mama.

4. Jemal A, Murria T, Ward E, Samuel A, Tiwari R, Ghafoor A, Fever E, Thun M. Cancer Statistics 2005. CA Cancer I Clin. 2005;55:10-30.

5. Dimo T, Rakotonirina S, Tan P, Azay J, Dongo E, Cros G. Leaf methanol extract of Bidens pilosa prevents and attenuates the hypertension induced by highfructose diet inWistar rats. J Ethnopharmacol. 2002;83:183-91.

6. Tan P, Dimo T, Dongo E. Effects of methanol, cyclohexane and methylene chloride extracts of Bidens pilosa on various gastric ulcer models in rats. J Ethnopharmacol. 2000;73:415-21.

7. Chih H, Lin C, Tang K. The hepatoprotective effects of Taiwan folk medicine Ham-hong-chho in rats. Am J Chin Med. 1996;24:231-40.

8. Jager A, Hutchings $A$, vanStaden J. Screening of Zulu medicinal plants for prostaglandin-synthesis inhibitors. Journal of Ethnopharmacol. 1996; 52: 95-100.

9. Chang J, Chiang L, Chen C, Liu L, Wang K, Lin C. Antileukemic activity of Bidens pilosa $L$. var. minor (Blume) Sherff and Houttuynia cordata Thunb. Am J Chin Med. 2001;29:303-12.

10. Rabe T, vanStaden J. Antibacterial activity of South African plants used for medicinal purposes J Ethnopharmacol. 1997;56:81-7.

11. Arroyo J, Bonilla $P$, Ráez E, Suárez S, Palomino $\mathrm{R}$, Terán $\mathrm{S}$, et al. Compuestos fenólicos de la fracción metanólica de Bidens pilosa, sobre la neoplasia gástrica, inducida en ratas. An Fac Med. 2007;68(2):105-12.

12. Domínguez J. Contribuciones a la Materia Médica Argentina, Bs. As., Ed. Peuser. 1928:427-33.

13. Mabry T, Markham K, Thomas M. The Systematic Identification of Flavonoids. New York. Springer Verlag; 1970:350-4.

14. Lock de Ugaz, 0. Investigación fítoquímica; métodos en el estudio de productos naturales, 2da edición. Fondo Editorial Pontificia Universidad Católica del Lima- Perú. 1994:15-20.

15. Barroso A, Muranaka E, Mori J, Pelizon C, Iriya K, Giocondo G, Aristodemo J. Induction of experimental mammary carcinogénesis in rats with 7,12-Dimethylbenz (a) anthracene. Rev Hosp Clin Fac Med. 2004;59(5):257-61.

16. Schmidt W. The micronucleus test. Mutat Res. 1975;9:31-9.

17. Buege J, Aust S. Microsomal lipid peroxidation. Methods Enzymol. 1978;52:302-10.

18. Suárez S. Detoxificación hepática y defensa antioxidante por efecto de xenobióticos alimentarios. Tesis para optar al Grado Académico de Magíster en Bioquímica. Lima: Facultad de Medicina, UNMSM; 1995.

19. Weimer T, Reddy A, Harttig U, Alexander D, Stamm C, Miller M, et al. Influence of B-naphthoflavone on 7,12-dimethylbenz(a)anthracene metabolism, DNA adduction, and tumorigenicity in rainbow trout. Toxicol Sci. 2000;57:217-28.

20.Schnitz AR, O'Connor JM. In vivo DNA/RNA adduction of 7,12-dimethylbenz(a)anthracene (DMBA) and benzo(a)pyrene (BaP) in the liver of rainbow trout (Oncorhynchus mykiss). J Env Path Tox Oncol. 1992;11:229-33.

21. Morrison VM, Burnett AK, Forrester LM, Wolf CR, Craft JA. The contribution of specific cytochromes P450 in the metabolism of 7,12-dimethylbenz(a) anthracene in rat and human liver microsomal membranes. Chem Biol Interact. 1991;79:179-96.

22. Tanaka T, Makita H, Ohnishi M, Hirose Y, Wang A, Mori $\mathrm{H}$, et al. Chemoprevention of 4-nitroquinoline 1-oxide-induced oral carcinogenesis by dietary curcumin and hesperidin: Comparison with the protective effect of $\beta$-carotene. Cancer Res. 1994;54:4653-9.
23. Kim HP, Son KH, Chang HW, Kang SS. Antiinflammatory plant flavonoids and cellular action mechanisms. J Pharmacol Sci. 2004;96:229-45.

24. Sakata K, Hirose Y, Qiao Z, Tanaka T, Mori H. Inhibition of inducible isoforms of cyclooxygenase and nitric oxide synthase by flavonoid hesperidin in mouse macrophage cell line. Cancer Lett. 2003;199:139-45.

25. Russo J, Gusterson B, Rogers A, et al. Biology of disease: comparative study of human and rat mammary tumorigenesis. Lab Invest. 1990;62:244-57.

26. Clarke R. Issues in experimental design and endpoint analysis in the study of experimental cytotoxic agents in vivo in breast cancer and other models. Breast Cancer Res Treat. 1997;46:25578.

27. Wang Y, Chanc F, Cheng Sh, Leung L. The plant polyphenol butein inhibits testosterone-induced proliferation in breast cancer cells expressing aromatase. Life Sci. 2005;77:39-51.

28. Finkel T, Holbrook J. Oxidants, oxidative stress and the biology of ageing. Nature. 2000;408:23947.

29. Esposito E, Rotilio D, Di Matteo, V., Di Giulio C, Algeri, A. review of specific specific dietary antioxidants and the effects on biochemical mechanisms related to neurodegenerative processes. Neurobiol Aging. 2002;23:719-35.

30. Jang HS, Kook SH, Son YO, Kim JG, Jeon YM, Jang YS, Choi KC, Kim J, Han SK, Lee KY, Park BK, Cho NP, Lee JC. Flavonoids purified from Rhus verniciflua Stokes actively inhibit cell growth and induce apoptosis in human osteosarcoma cells. Biochim Biophys Acta. 2005;1726(3):309-16.

31.Deog K, Liping L, Weimin G, Mohsen M. Chemical structure of flavonols in relation to modulation of angiogenesis and immune-endothelial cell adhesion. J Nutritional Biochem. 2005;17:16576.

32. Rietjens I, Boersma M, Van der Woude H, Jeurissen S, Schutte M, Alink G. Flavonoids and alkenylbenzenes: Mechanisms of mutagenic action and carcinogenic risk. Mutat Res. 2005;574:12438.

33. Instituto Nacional de Enfermedades Neoplásicas. http://www.inen.sld.pe/intranet/ estadepidemiologicos.htm (12 abril 2007).

Manuscrito recibido el 8 de junio de 2010 y aceptado para publicación el 28 de setiembre de 2010.

\section{Correspondencia:}

Dr. Jorge Luis Arroyo Acevedo

Sección de Farmacología

Facultad de Medicina, UNMSM.

Av. Grau 750. Lima 1, Perú.

Correo-e: jorgeluis_arroyoacevedo@yahoo.es 